\title{
Editorial
}

\section{Cognition in psychiatry}

\author{
Sonia Acuna-Vargas, MPsych; Florence Thibaut, MD, PhD - Editor in chief
}

Cognition has attracted a growing interest in psychiatry. Since the 1990s, cognition as a whole has become an important determinant in the outcome of psychosis. Despite recent progress in the genetics of cognition, the development of new pharmacological compounds in order to improve cognition has not progressed as quickly. This issue will review and discuss the main areas of clinical and basic research in this domain.

Keywords: cognition; psychiatric disorder; psychosis; genetics

A quick Medline search using the keywords "cognition" and "psychiatry" generated about 40000 records. Numerous publications are related to memory and/or executive functioning in psychotic disorders, or to aging and dementia. Cognitive performance in clinical neuropsychology typically refers to assessment of cognitive domains of functioning. Originally, these dom ains were linked to specific brain areas where processes were supposed to take place. More recently, circuit-based conceptions focused on activation of different brain regions and their potential interactions. Moreover, brain imaging helped to understand how brain structures might be involved in normal and pathological cognition. Cognitive assessment was currently used to identify patterns of performance/impairments across domains as markers of functional deficits, which could becom e treatm ent targets in populations with brain damage, degenerative diseases and psychiatric conditions. Finally, metacognition (ie, "knowledge and regulation of cognition") is a concept which has been developed since 1970. Flavell' distinguished several aspects of "knowledge": a declarative or person knowledge (understanding one's own abilities); a procedural or task knowledge; and a strategy knowledge (one's ability to use strategies to learn information and adapt these strategies to novelty). The term "regulation" was used to describe how people monitor and assess their knowledge. Motivation plays also an important role in metacognition. The term "cognitive," as used in most contemporary psychotherapeutic interventions, refers to a metacognitive approach aimed at modifying inappropriate thoughts and beliefs.
Psychiatric populations were usually classified according to the prominent clinical symptoms observed, independently of the cognitive dysfunctions measured. Yet, these latter dysfunctions may overlap among psychiatric disorders. A better understanding of these common cognitive dysfunctions may contribute to identifying the brain mechanisms involved in these disorders.

Moreover, psychotic symptoms are observed in primary psychotic disorders (eg, schizophrenia) as well as in affective psychoses (eg, bipolar disorder with psychotic features); they can also occur in certain somatic disorders or, in some patients exposed to certain medications/substances or to alcohol. Beyond clinical sym ptom $\mathrm{s}$, a significant $\mathrm{m}$ ajority of patients with psychosis also present impaired cognition. Since the 1990 s, there has been a growing interest and recognition in cognition as an important determinant in the outcome of psychosis. Thus, the prevalence, profile and $m$ agnitude, the developmental course and the longitudinal stability of cognitive impairment are major areas of research to target treatments, which can improve functional outcome in these populations. McCleery and Nuechterlein (p 239) as well as Robbins et al (p 301) review this topic in this issue. Moreover, Harvey, in this issue (p 227), reviews the assessment of major cognitive domains and comments on whether each dom ain fits into a top-down or bottom -up structure (com plex vs basic domains). In addition, the intrinsic validity of these tests was raised in psychiatric populations (which differ from the patients with specific regional brain lesions in whom these

Author affiliations: University Hospital Cochin - site Tarnier, Paris, France (Florence Thibaut, Sonia Acuna-Vargas), Faculty of Medicine Paris Descartes (Paris University), INSERM U1266, Institute of Psychiatry and Neuroscience, Paris, France (Florence Thibaut). Address for correspondence: florence.thibaut@aphp.fr 


\section{Editorial}

Cognition in psychiatry - Acuna-Vargas, Thibaut

tests were originally validated). As deficits in higher-order executive functions and decision-making are significant predictors of relapse, cognitive training, remediation, and pharmacological approaches have the potential to rescue cognition. To date, cognitive rehabilitation has focused on cognitive enhancing pharmacological agents and cognitive training (CT). CT has shown efficacy, while glutam atergic and cholinergic pharmaceutical compounds demonstrated small improvements in working memory and overall cognition. Physical exercise and neurostimulation are new areas of investigation, which can be combined with other approaches (eg, with CT) to improve cognition (see Moritz et al [p 309] and Schm itt et al [p 261] in this issue). In addition, the benefit of not only exercise, but also physical activity in general, is linked to improvement of neural plasticity and neurogenesis.

Furtherm ore, whereas cognitive deficits can be considered as symptoms when "cold cognition" domains are impaired (attention, memory, working memory, etc), they can also be understood to interact with emotional or motivational factors in the case of "hot cognition" impairments (decision making, impulsivity, social cognition, etc). Until recently, this latter area was insufficiently studied, especially in psychotic disorders and autism, where these deficits are associated with $m$ ajor social adjustm ent difficulties. Blair ( $p$ 291) discusses in this issue two forms of dysfunctional neurocognition that increase the risk of psychopathology. Firstly, a reduced emotional responsiveness, empathy, moral judgment, and immoral behavior may increase the risk for aggressiveness in adolescents and adults. Secondly, reduced reward responsiveness, including to drug cues in adolescents with clinically antisocial behavior, may increase the risk for psychopathy.

More broadly, Verdejo-Garcia et al, in this issue (p 281) summarize knowledge about substance-use disorders including alcohol, stimulants, and opioids related to cognitive deficits.

Kühn et al, in this issue ( $p$ 319) review video gaming and its involvement in cognition as well as in functional and struc- tural changes in brain regions. Among observed effects, visuospatial cognition and attention seem to be enhanced; this might be able to be used in cognitive remediation of certain psychiatric disorders.

Ongoing research focused on the association of cognitive function and presynaptic proteins, which could become a target for new drugs, taking into consideration that presynaptic plasticity contributes to cognitive function evolution during aging (see Honer et al in this issue, p 271).

Finally, according to recent studies, single-nucleotide polymorphism (SNP)-based estimates of heritability for general cognitive function were approximately $20 \%$ to $30 \%$; these estimates might increase to $50 \%$ when family-based designs were used (due to potential contributions of rarer SNPs). ${ }^{2}$ Davies et al, ${ }^{3}$ analyzing more than 300000 individuals of European ancestry, aged between 16 and 102 years, reported 148 genome-wide significant loci for general cognitive function. In this study, a general cognitive function component phenotype was constructed from a number of cognitive tasks. Using polygenic scoring, $4.31 \%$ of the general cognitive function variance was predicted in an independent sample. In Davies' study, variants associated with neurodegenerative as well as neurodevelopmental disorders, physical and psychiatric illnesses, or brain structures, neural and cell development were reported. In parallel, most of the candidate genes involved in major psychiatric disorders were also involved in core neurodevelopmental processes which are strongly associated with synaptic plasticity, brain circuit formation, and, ultimately, higher-order cognitive performance. Yet, the development of new pharmacological compounds in order to improve cognition has not progressed as quickly as genetics. Both the lack of genetically validated targets and of mechanisms involved and, the almost exclusive focus of preclinical research and industrial drug discovery on aminergic signaling, have contributed to this delay. Stephan et al, in this issue ( $\mathrm{p}$ 249), will discuss new concepts and future potential of translational research on cognition in psychiatry.

\section{References}

1. Flavell JH. Metacognition and cognitive monitoring: a new area of cognitive-developmental inquiry. Am Psychol. 1979;34(10):906-911.

2. Hill WD, Arslan RC, Xia C, et al. Genomic analysis of family data reveals additional genetic effects on intelligence and personality. Mol Psychiatry. 2018;23(12):2347-2362. doi:10.1038/s41380-017-0005-1.

3. Davies G, Lam M, Harris SE, et al. Study of 300,486 individuals identifies 148 independent genetic loci influencing general cognitive function. Nat Commun. 2018;9:2098. doi:10.1038/s41467-018-04362-x. 